\title{
Ecología trófica y rasgos ecomorfológicos del pez Triportheus magdalenae (Characiformes: Triportheidae) en el embalse El Guájaro, río Magdalena, Colombia
}

\author{
Jenny J. Morales ${ }^{1} \&$ Carlos A. García-Alzate ${ }^{2 *}$ \\ 1. Pós-Graduação em Ecologia e Conservação da Biodiversidade, Instituto de Biociências, Universidade Federal de \\ Mato Grosso (UFMT), Cuiabá-MT, Brasil; jennymoralesp@gmail.com \\ 2. Programa de Biología, Colecciones Científicas Museo de Biología, Facultad de Ciencias Básicas, Universidad del \\ Atlántico; Barranquilla, Colombia; carlosgarciaa@mail.uniatlantico.edu.co \\ * Correspondencia
}

Recibido 26-III-2018. Corregido 18-V-2018. Aceptado 19-VI-2018.

\begin{abstract}
Trophic ecology and ecomorphological traits of the fish Triportheus magdalenae (Characiformes: Triportheidae) in the EI Guájaro reservoir, Magdalena River, Colombia. This study integrated morphological characteristics and trophic ecology of Triportheus magdalenae in the El Guájaro reservoir, Atlántico, lower Magdalena River Basin, Colombia, based on the analysis of stomach contents and ecomorphological attributes. Five samplings were made between September 2014 and February 2016 considering each phase of the flood pulse. The fish were sampled using traditional fishing gear such as nets and trawls. The index of food importance (IAi \%) was used to establish the dietary habits of the species, and the variation of the diet at interspecific level, and the vacuity coefficient (CV \%) was also calculated. The Levins index standardized by Hurlbert $\left(\mathrm{B}_{\mathrm{A}}\right)$ was used to evaluate the amplitude between sizes, sex and hydrological phases. The oropharyngeal structures and the digestive tract were characterized, which together with morphological measurements, allowed and analyze 11 ecomorphological traits related to the use of habitat, such as position in the water column, and the exploitation of a particular trophic resource. The relationship between the ecomorphological traits and the diet of the species was analyzed by canonical correspondence analysis (CCA). We analyzed 89 individuals (34-124 $\mathrm{mm}$ SL). Triportheus magdalenae was classified as carnivore/zooplanktivore, and has a narrow trophic niche $\left(\mathrm{B}_{\mathrm{A}}=0.12\right)$. Showed a high exploitation of zooplankton (95.9\% IAi), mostly Cladocera (Ceriodaphnia sp.) and Copepoda (Notodiaptomus sp. and Thermocyclops sp). The youngest juveniles (34-190 mm SL) also consumed allochthonous insects ( $3.4 \%$ IAi). The ecomorphological traits that contributed the most to this trophic specialization were the relative length of the gillrakers (LRBE). The number of gillrakers (NBE), which varied significantly from the smaller to the larger ones, increase in both number as well as in length as individuals grow, which explains the strongly marked degree of specialization on zooplankton in adults, since they present a greater degree of efficiency in the process of filtering. The vacuity coefficient was high (54\%), which could be associated with a decrease in the density of zooplankton populations, especially during the time of lower waters strongly influenced by the El Niño event, however the observed diet ( 22 items food) corresponded to 80 $\%$ of the diet estimated (28 estimated food items). Rev. Biol. Trop. 66(3): 1208-1222. Epub 2018 September 01.
\end{abstract}

Key words: diet; ecomorphological traits; endemic fish; diversity.

Los estudios ecológicos permiten conocer además de la diversidad de especies de un ecosistema, las interacciones bióticas interespecíficas e intraespecíficas. Además, el conocimiento de la dieta de las especies permite describir tales interacciones y la forma en la que se encuentran organizadas las especies al interior de un nicho ecológico en su eje trófico, con base en el alimento que consumen. Asimismo, permiten explorar la amplitud del nicho trófico mediante la cuantificación de la variación en la utilización de los recursos (Valverde, Cano-Santana, Meave, \& Carabias, 2005). Para estudios de ecología alimentaria de los peces, 
es necesario abordar interrogantes como: ¿qué consumen?, ¿dónde?, ¿cómo lo hacen? y ¿cuál es la magnitud de la variación de la dieta con relación al tamaño? Considerar también las variaciones entre sexos y el momento hidrológico, dado que la dinámica entre la disponibilidad de alimento y la amplitud del nicho trófico de las especies tropicales están sujetas a los pulsos de inundación (Abelha, Agostinho, \& Goulart, 2001).

En investigaciones ecológicas donde se estudia la estructura trófica relacionada con rasgos ecomorfológicos en peces, se usan diversos métodos de análisis que permiten conocer las relaciones que se establecen entre el ambiente y los organismos. Entre ellos, estudios cuantitativos y cualitativos del contenido estomacal (Hyslop, 1980), y estudios de diversos rasgos morfológicos que se encuentran estrechamente correlacionadas y permiten explicar la explotación de los recursos y lo cual depende en gran medida de la morfología interna (Pianka, 2000; Chen, 2002; Pouilly, Lino, Bretenoux, \& Rosales, 2003; Sánchez, Galvis, \& Victoriano, 2003; Zarate-Hernández, Aguirre-León, Ortiz-Burgos \& Castillo-Rivera, 2007). Este campo de estudio brinda conocimiento no solo de cuáles son los recursos consumidos sino también de cómo intervienen las adaptaciones morfológicas (Ebert \& Bizarro, 2007). Asimismo, la existencia de variabilidad en los rasgos morfológicos a nivel intraespecífico impulsa a la especialización trófica de las especies. Sin embargo, a pesar de tales variaciones, la mayoría de los trabajos se han centrado principalmente en las diferencias en los rasgos funcionales entre las especies, sin tener en cuenta los efectos potenciales de variaciones intraespecíficas (Zhao, Villéger, Lek, \& Cucherousset, 2014).

Triportheus magdalenae (Steindachner, 1878) es un pez endémico de la cuenca Magdalena con un alto valor como recurso pesquero en la región, además de su papel ecológico en los ecosistemas, ya que es un consumidor secundario (Rodríguez \& Rodríguez, 1976; Malabarba, 2004; Caraballo, 2009; JiménezSegura, Carvajal-Quintero, \& Aguirre, 2010; Mariguela, Roxo, Foresti, \& Oliveira, 2016). El objetivo de este estudio fue analizar las variaciones tróficas intraespecíficas de esta especie en el embalse El Guájaro-Atlántico Colombia, en donde estudiamos las relaciones entre la dieta y rasgos ecomorfológicos asociados a la explotación del alimento, principalmente a las características del branquio-cráneo.

\section{MATERIAL Y MÉTODOS}

Área de estudio: El área hidrográfica del Magdalena-Cauca (5701101 ha) alberga los embalses más grandes del país, y representan el $18 \%$ del total de humedales continentales a nivel nacional. El embalse El Guájaro, fue construido entre 1964 y 1965 por INCORA con el fin de captar agua y para los distritos de riego del sur del departamento del Atlántico (CRA, 2002). Se Ubica entre los $10^{\circ} 25^{\prime}-10^{\circ} 38^{\prime}$ $\mathrm{N} \& 75^{\circ} 00^{\prime}-75^{\circ} 08^{\prime} \mathrm{W}$, y de 3 a 13 m.s.n.m., a la ribera izquierda del Río Magdalena, al suroccidente del departamento de Atlántico, costa Caribe Colombiana. Actualmente el embalse ocupa una extensión de 16000 ha, donde se desarrollan principalmente actividades pesqueras, agrícolas, consumo humano, recreación y turismo, ubicándolo como el cuerpo de agua más importante de la región (CRA, 2002; Caraballo, 2009; García-Alzate, Moreno, \& De la Parra, 2016).

El embalse corresponde a áreas bajas del río Magdalena y se conecta con sus aguas en el extremo sur, por medio del Canal del Dique, se caracteriza por poseer gradientes limnológicos horizontales con concentraciones de mayor a menor en sentido Norte-Sur de la conductividad eléctrica, alcalinidad, dureza y $\mathrm{pH}$, especialmente en periodos de sequia (Villalón \& Vega, 2016; García-Alzate et al. 2016). Considerando la morfología del embalse, el aporte de agua y el comportamiento de las características limnológicas, se establecieron nueve estaciones de muestreo (E1 a E3 en la zona Norte, E4 a E6 en el Centro, y en el Sur de E7 a E9) (Fig. 1).

Diseño muestral: Se realizaron cinco salidas de campo (M1 a M5) entre septiembre 2014 y febrero 2016 con el propósito abordar 


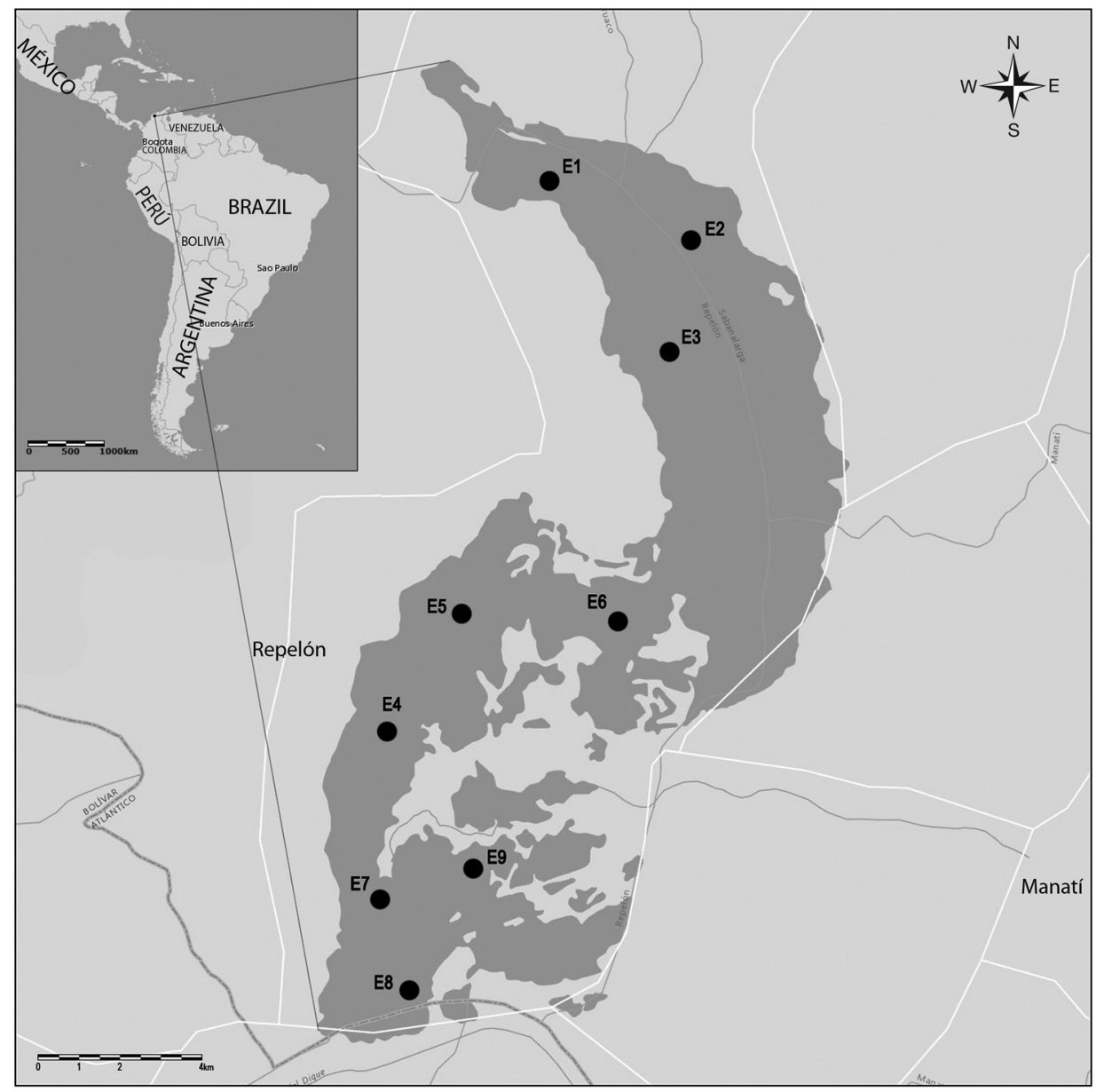

Fig. 1. Embalse El Guájaro (9 estaciones de muestreo: E1, E2, E3 en la zona Norte, E4, E5, E6 en el Central, y E7, E8, E9 en el Sur).

Fig. 1. E1 Guájaro Reservoir (9 sampling stations: E1, E2, E3 in the North, E4, E5, E6 in the Center, and E7, E8, E9 in the south).

cada momento del pulso de inundación: subida de aguas (M1 septiembre de 2014, $3.27 \mathrm{~m}$ de nivel), en aguas altas (M2 diciembre de 2014, $4.37 \mathrm{~m}$ ), retirada de aguas (M3 y M4 abril y junio 2015, $3.56 \mathrm{~m}$ y $3.18 \mathrm{~m}$ de nivel respectivamente), y aguas bajas fuertemente influenciado por el fenómeno El Niño 2015-II a 2016-I (M5 en febrero 2016, 2.46 m) (Fig. 2).

Las capturas de los peces se llevaron a cabo mediante el uso de atarrayas (ojos de malla de $1 \mathrm{~cm}$ y $2.5 \mathrm{~cm}$, con 3 y $5 \mathrm{~m}$ de diámetro, respectivamente) y redes de arrastre (ojos de malla de $0.5 \mathrm{~cm}$ y $1 \mathrm{~cm}, 3$ x 4 y 3 x $5 \mathrm{~m}$, respectivamente). Los ejemplares capturados fueron registrados y depositados en neveras de polietileno con hielo para su posterior análisis.

Trabajo de laboratorio: Los ejemplares recolectados, fueron medidos (con un calibrador digital; Longitud Estándar [LE mm]) y pesados (con una balanza digital de $0.01 \mathrm{~mm}$ de precisión $[\mathrm{g}])$. Seguidamente con base en la longitud estándar se clasificaron en intervalos de tallas, al utilizar la ecuación: $A=N / R ; R=$ $\sqrt{ }$; $\mathrm{N}=$ Max-Min; donde, $\mathrm{A}=$ amplitud de los intervalos, $\mathrm{R}=$ número de intervalos, $\mathrm{n}=$ número de observaciones, $\mathrm{Max}=$ valor máximo, $\mathrm{Min}$ = valor mínimo (Se sigue a García-Alzate, 


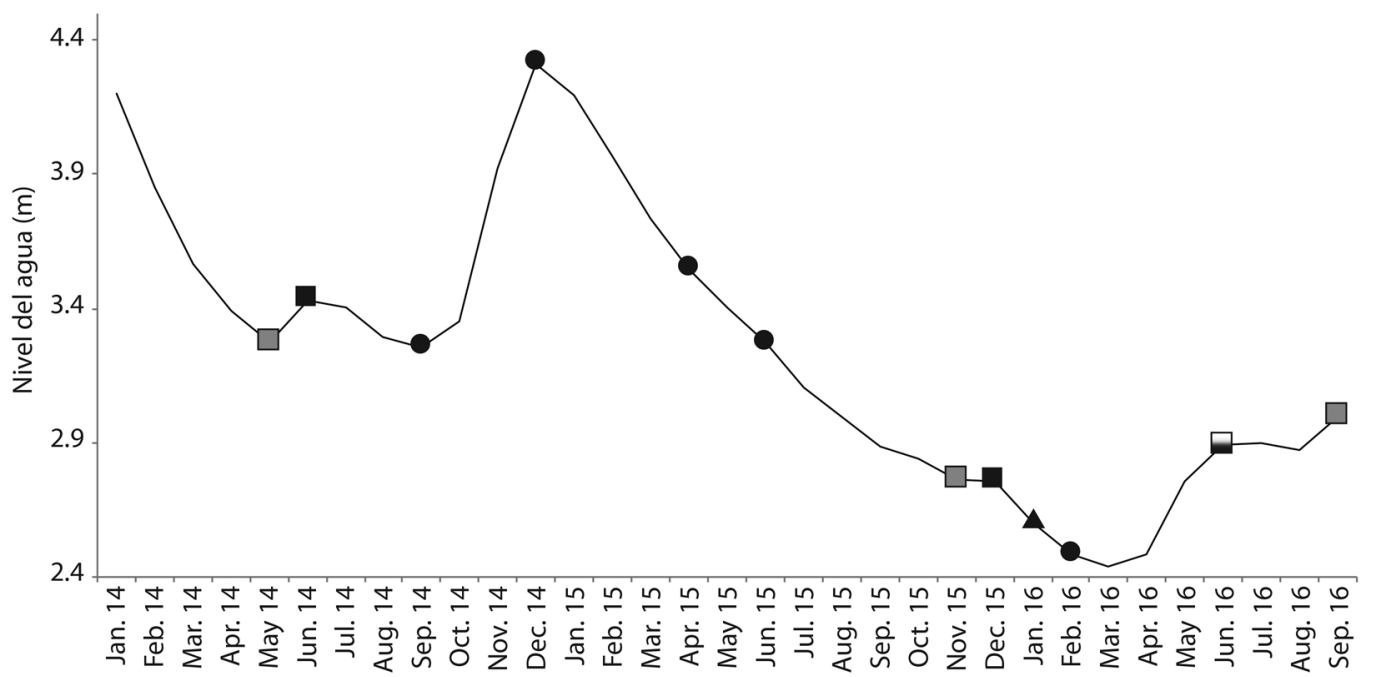

Fig. 2. Pulso de inundación del embalse El Guájaro entre 2014 y 2016, modificado de García-Alzate et al. (2016) y estación AcuaCultivos El Guájaro en La Peña. $\square=$ compuertas abiertas; $\boldsymbol{\square}=$ compuertas cerradas; $\boldsymbol{0}=$ muestreos $1,2,3,4$ y 5 de izquierda a derecha; $\boldsymbol{\Delta}$ = inicio de bombeo desde el canal del Dique; $\square=$ apertura y cierre de las compuertas.

Fig. 2.Flood pulse The Guájaro reservoir between 2014 and 2016, modified García-Alzate et al. (2016) and AcuaCultivos The Guájaro station in La Peña. $\square=$ open gates; $\boldsymbol{\square}=$ closed gates; $\boldsymbol{\bullet}=$ samples 1, 2, 3, 4 and 5 from left to right; $\boldsymbol{\Lambda}=$ start of pumping from the channel of the dam; $\square=$ opening and closing of the gates.

Román-Valencia \& Barrero, 2012; Morales \& García-Alzate, 2016; Ibarra-Trujillo \& GarcíaAlzate, 2017).

Posteriormente, mediante incisión longitudinal en la zona uroventral se extrajo el tracto digestivo de todos los ejemplares el cual fue pesado, medido, rotulado y conservado en alcohol al $70 \%$ para el posterior análisis. Los ejemplares fueron fijados en formol al $10 \%$ y depositados en la colección de referencia científica de la Universidad del Atlántico reconocida por el IAvH con número de registro UARC-132 (Morales \& García-Alzate, 2016).

Se realizó una caracterización del tracto digestivo como longitud; ancho y forma del estómago, y longitud del intestino con la ayuda de un calibrador digital $(0.01 \mathrm{~mm}$ de precisión). Asimismo, estructuras bucofaríngeas como el número y longitud de branquiespinas, y el número y forma de los dientes. Los cuales fueron contados y medidos por cada intervalo de talla, con la ayuda del método de clareado y teñido (Taylor, 1969), bajo un microscopio estereoscópico.
Se midieron 11 rasgos ecomorfológicos relacionados con las funciones vitales tales como la posición de la especie en la columna de agua, explotación del recurso trófico, hábito trófico y tamaño de las presas (Zhao et al., 2014) (Cuadro 1), basados las publicaciones de Gatz (1979), Watson \& Balon (1984), Balon, Crawford \& Lelek (1986), Pouilly et al. (2003); Teixeira \& Bennemann (2007), Fernández, Ferriz, Bentos \& López (2012) y Ramírez, Lee \& Mojica (2015). Los datos de los rasgos ecomorfológicos se analizaron con relación a la Longitud Estándar (LE) con el fin de reducir el efecto alométrico y disminuir el efecto de las diferencias en tamaño corporal (Winemiller, 1991).

Análisis de datos: Para la determinación de los ítems alimentarios el contenido estomacal fue depositado en cajas Petri y examinado bajo el microscopio estereoscopio. La identificación se realizó con la ayuda de claves específicas hasta la categoría taxonómica más baja posible (Roldán, 1996; Ramírez, 2000; Fernando, 2002 y Domínguez \& Fernández, 2009). 
CUADRO 1

Rasgos ecomorfológicos, código y descripción asociados con tamaño de presa, posición del pez en la columna de agua, explotación del recurso trófico y hábito trófico

TABLE 1

Ecomorphological traits, code and description associated with the size of the dams, position of the fish in the water column, exploitation of the trophic resource and trophic habit

\begin{tabular}{|c|c|c|c|}
\hline ASOCIADOS A: & RASGO & CÓDIGO & DESCRIPCIÓN \\
\hline \multirow{3}{*}{$\begin{array}{l}\text { Tamaño de } \\
\text { las presas }\end{array}$} & Longitud estándar & $\mathrm{LE}$ & Distancia de la punta del hocico hasta la última vértebra. \\
\hline & $\begin{array}{l}\text { Longitud relativa } \\
\text { de la cabeza }\end{array}$ & LRC & $\begin{array}{l}\text { Relaciona la distancia de la punta del hocico hasta el margen } \\
\text { posterior del opérculo y es estandarizada al dividirla por la } \\
\text { longitud estándar (LE). }\end{array}$ \\
\hline & $\begin{array}{l}\text { Altura relativa } \\
\text { de la boca }\end{array}$ & $\mathrm{ARB}$ & $\begin{array}{l}\text { Es la relación entre la altura máxima de la boca sobre la } \\
\text { longitud estándar. }\end{array}$ \\
\hline \multirow[t]{4}{*}{$\begin{array}{l}\text { Posición del pez } \\
\text { en la columna } \\
\text { de agua (vertical) }\end{array}$} & $\begin{array}{l}\text { Posición } \\
\text { de la boca }\end{array}$ & $\mathrm{PB}$ & $\begin{array}{l}\text { Es la orientación de la boca: Posición dorsal }=1 \text {; Terminal }= \\
2 \text {; Ventral =3. Basados en la clasificación de Watson \& Balon } \\
\text { (1984) y Hugueny \& Pouilly (1999): indica la posición de los } \\
\text { alimentos con respecto al pez. }\end{array}$ \\
\hline & $\begin{array}{l}\text { Diámetro relativo } \\
\text { del ojo }\end{array}$ & DRO & $\begin{array}{l}\text { Es la relación del diámetro horizontal del ojo estandarizado al } \\
\text { dividirlo entre la LE. }\end{array}$ \\
\hline & $\begin{array}{l}\text { Posición relativa } \\
\text { de los ojos }\end{array}$ & PRO & $\begin{array}{l}\text { Es la relación entre la distancia vertical desde el centro del ojo } \\
\text { hasta el límite inferior de la cabeza dividida entre la altura total } \\
\text { de la cabeza medida a nivel de los ojos. }\end{array}$ \\
\hline & $\begin{array}{l}\text { Configuración de } \\
\text { la aleta pectoral } \\
\text { izquierda }\end{array}$ & CAP & $\begin{array}{l}\text { Es el producto de relación entre la longitud y ancho máximo de } \\
\text { la aleta pectoral estandariza con la LE. }\end{array}$ \\
\hline \multirow[t]{3}{*}{$\begin{array}{l}\text { Explotación del } \\
\text { recurso trófico }\end{array}$} & Dientes & $\mathrm{D}$ & $\begin{array}{l}\text { se combinaron en un código basados en el número y la forma en } \\
\text { el pre-maxilares, maxilar y dentario para describir el modo de } \\
\text { la ingesta: } 0=\text { ausencia de dientes (asociado con la función de } \\
\text { succión o aprovechando las presas pasivas). } 1 \text { = pocos dientes } \\
<50 \text { de forma cónica o multicuspidal (asociados al morder o } \\
\text { desgarrar). } 2=\text { numerosos dientes }>50 \text { filiformes (asociados } \\
\text { con la actividad de raspado). } 3=\text { banda de numerosos dientes } \\
\text { (asociado con succión aprovechando las presas activas). }\end{array}$ \\
\hline & $\begin{array}{l}\text { Número de } \\
\text { branquiespinas en el } \\
\text { primer arco branquial } \\
\text { izquierdo }\end{array}$ & NBE & $\begin{array}{l}\text { Es la suma de las branquiespinas en la parte superior e inferior } \\
\text { del primer arco branquial. }\end{array}$ \\
\hline & $\begin{array}{l}\text { Longitud relativa de } \\
\text { branquiespinas en el } \\
\text { primer arco branquial } \\
\text { izquierdo }\end{array}$ & LRBE & $\begin{array}{l}\text { Es la relación de la longitud media de la profundidad de corte } \\
\text { de las branquiespinas del primer arco branquial sobre la LE. }\end{array}$ \\
\hline Hábito trófico & $\begin{array}{l}\text { Longitud relativa } \\
\text { del intestino }\end{array}$ & LRI & Es la relación entre la longitud total del intestino sobre la LE. \\
\hline
\end{tabular}

Posteriormente, se siguió a Morales \& García-Alzate (2016), con el uso del Coeficiente de Vacuidad (CV \%), el cual expresa en porcentaje el número de individuos capturados que no tenían contenido en sus estómagos. Seguidamente, para evaluar la suficiencia del tamaño de la muestra de los estómagos con alimento, se realizó una curva de acumulación de presas o ítems con el usó del índice no paramétrico Chao2 para estimar la riqueza específica de la dieta consumida, basados en datos de presencia/ausencia. 
Para establecer el hábito trófico de la especie y evaluar la variación intraespecífica de la dieta se aplicó el Índice Alimentario (IAi \%) (Kawakami \& Vazzoler, 1980). Los datos fueron sometidos a pruebas de normalidad y homocedasticidad, posteriormente se aplicó una prueba de Kruskal-Wallis con el fin de determinar diferencias en la dieta a nivel temporal e intraespecíficas, es decir, entre sexos, y ontogénico de la población.

Se determinó la amplitud de nicho trófico con el índice de Levins estandarizado (Hurlbert 1978) $\left(B_{A}\right)$ con base en Krebs (2014), la escala de valores resultantes son entre 0 (mínima amplitud), y 1 (máxima amplitud), cuando son menores a 0.6 indica estrecha amplitud. Asimismo, en el análisis de similitud trófico, se aplicó el índice de Morisita simplificado (Krebs, 2014), cuyos valores varían entre 0 y 1 , donde 0 (ausencia de similitud) y 1 (máxima similitud) trófica. Se calculó la amplitud y similitud trófica entre intervalos de tallas, sexos y momentos del pulso de inundación, basados en los valores del índice alimentario (IAi).

Se realizaron análisis de posición central y de dispersión para observar la tendencia de los rasgos ecomorfológicos, pruebas de normalidad (Shapiro-Wilk, $\mathrm{n}=11$ ) y un ANDEVA con el fin de determinar las diferencias intraespecíficas de los rasgos ecomorfológicos. Las relaciones entre la dieta y de los rasgos ecomorfológicos se estudiaron mediante el análisis de correspondencia canónica (ACC), contrastando los rasgos ecomorfológicos en cada intervalo de talla y las categorías alimenticias registradas (IAi \%), donde los rasgos ecomorfológicos se constituyen como variables explicativas de la alimentación (Ter-Braak, 1986). La significación estadística se estimó con 1000 permutaciones. Todas las pruebas fueron realizadas con la ayuda de los programas Microsoft Excel 2008 y Software PAST (versión 3.1.4) (Hammer, 2016).

\section{RESULTADOS}

Se recolectaron 89 individuos con un peso medio de $44 \mathrm{~g}$ ( 0.5 y $105 \mathrm{~g})$, y tamaño medio de
147 mm ( \pm 27 SD) LE (34 y 190 mm), los más frecuentes fueron de $150 \mathrm{~mm}, 170 \mathrm{~mm}$ y 180 $\mathrm{mm}$ de LE. Se establecieron seis intervalos de talla: I correspondió a individuos entre $34 \mathrm{~mm}$ a $64 \mathrm{~mm}$ LE, II entre 64.1 a $94 \mathrm{~mm}$ LE, III de 94.1 a $124 \mathrm{~mm}$ LE, IV entre 124.1 a $154 \mathrm{~mm}$ LE, V de 154.1 a $184 \mathrm{~mm}$ LE, y VI de 184.1 a $214 \mathrm{~mm}$ LE. La especie no fue capturada en zona Norte (E1, E2 y E3) del embalse, mientras que en la zona Centro se llevó a cabo el $37 \%$ de las capturas y en el Sur el $63 \%$ (Cuadro 2).

En la dieta de T. magdalenae se registraron 22 ítems alimentarios, de los cuales 11 fueron de origen animal especialmente zooplancton e insectos, y 10 de origen vegetal principalmente fitoplancton (Cuadro 3). El coeficiente de vacuidad fue de $54 \%$ equivalente a 48 estómagos vacíos. El estimador de acumulación de ítems alimentarios, indicó que la dieta equivale al $80 \%$ de los ítems estimados (Chao2: 28 ítems alimentarios vs 22 ítems observados). La especie mostró hábitos tróficos especialistas $\left(\mathrm{B}_{\mathrm{A}}=0.12\right)$ zooplanctófago, puesto que los microcrustáceos fueron los más importante en la dieta (12.37\% IAi), principalmente Copépoda (Notodiaptomus sp.: 37.7 \% IAi; Thermocyclops sp.: $13.2 \%$ IAi) y Cladócera (Ceriodaphnia sp.: 32.97 \% IAi), seguido de Insectos (3.37 \% IAi) especialmente alóctonos. Mientras que otros componentes como microalgas, ictioplancton (0.07\% IAi), escamas ( 0.13 $\%$ IAi), material vegetal ( $0.51 \%$ IAi; semillas y fibra vegetal) y detritus (0.02\% IAi) fueron considerados como alimentos accidentales.

Variación de la dieta entre juveniles y adultos: se detectaron diferencias significativas de la dieta entre tallas $(\mathrm{K}-\mathrm{W}, \mathrm{p}=0.037)$, dado que los juveniles $\left[\mathrm{I}=0 \mathrm{~B}_{\mathrm{A}}\right.$; II $=0.07 \mathrm{~B}_{\mathrm{A}}$; $\left.\mathrm{III}=0.11 \mathrm{~B}_{\mathrm{A}}\right]$ tuvieron hábitos zooplanctófagos seguido por el consumo de insectos; a excepción de los individuos de la talla I que consumieron exclusivamente insectos (alóctonos), y la talla III incluyó además de insectos (5.3\% IAi), Copépoda (5.7\% IAi), mientras que los adultos [IV $=0.12 \mathrm{~B}_{\mathrm{A}} ; \mathrm{V}=0.11 \mathrm{~B}_{\mathrm{A}} ; \mathrm{VI}=0.10$ $B_{A}$ ] solo tuvieron hábitos zooplanctófagos, las tallas IV y V incluyeron larvas de insectos en 


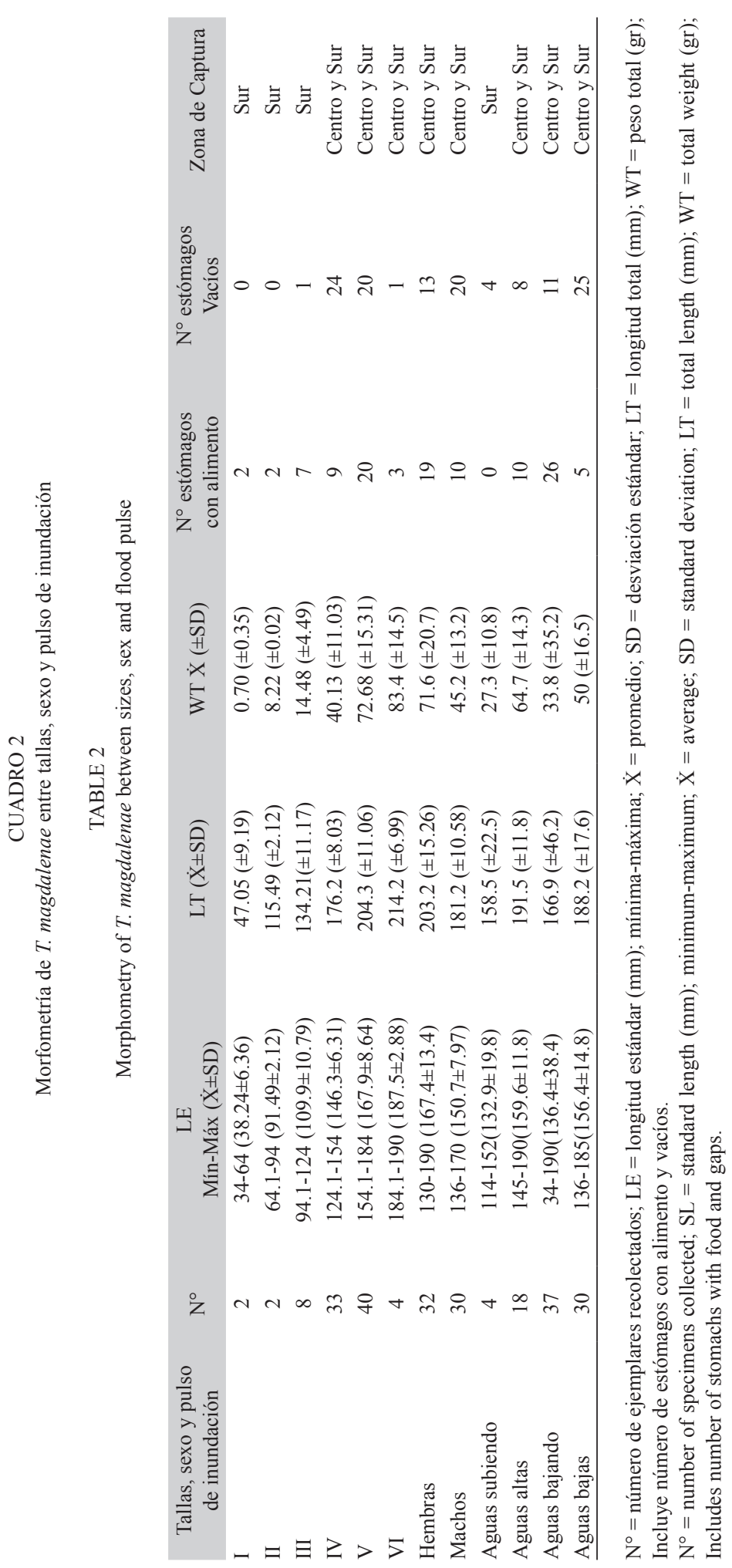


CUADRO 3

Dieta de T. magdalenae $(\mathrm{n}=89)$ en el embalse El Guájaro

TABLE 3

Diet of T. magdalenae $(\mathrm{n}=89)$ in the reservoir El Guájaro

\begin{tabular}{|c|c|c|c|c|}
\hline & Division / Phylum & Orden & Ítems & $\mathrm{IAi} \%$ \\
\hline \multirow[t]{8}{*}{ Fitoplancton } & Cyanobacteria & Nostocales & Dolichospermum sp. & 0 \\
\hline & & Oscillatoriales & Oscillatoria sp. & 0 \\
\hline & Cyanophyta & & Phormidium sp. & 0 \\
\hline & Chlorophyta & Chlorellales & Planctonema sp. & 0 \\
\hline & Bacillariophyta & Fragilariales & Synedra goulardii & 0 \\
\hline & & Aulacoseirales & Aulacoseira granulata & 0 \\
\hline & Heterokontophyta & Naviculales & Navicula sp. & 0 \\
\hline & & & Pinnularia sp. & 0 \\
\hline \multirow[t]{7}{*}{ Zooplancton } & Rotifera & Ploima & Keratella sp. & 0 \\
\hline & & & Trichocerca sp. & 0.02 \\
\hline & & & Brachionus sp. & 0.17 \\
\hline & Arthropoda- Crustáceos & Cladocera & Ceriodaphnia sp. & 32.97 \\
\hline & & Calanoida & Notodiaptomus sp. & 37.67 \\
\hline & & Cyclopoida & Thermocyclops sp. & 13.24 \\
\hline & & Rest. microcrustaceos & Rest. microcrustaceos & 11.82 \\
\hline \multirow[t]{2}{*}{ Macroinvertebrados } & Arthropoda-Insecta & Diptera & Diptera (L) & 0.06 \\
\hline & & Rest. insectos & Rest. insectos & 3.31 \\
\hline \multirow[t]{2}{*}{ Pisces-Osteichthyes } & Chordata-Vertebrata & Ictioplancton & Ictioplancton (L) & 0.07 \\
\hline & & Escamas & Escamas & 0.13 \\
\hline \multirow[t]{2}{*}{ Embryophyta } & Spermatophyta & Mat. Vegetal & Mat. Vegetal & 0.22 \\
\hline & & Semillas & Semillas & 029 \\
\hline Otros & Materia Orgánica & Detritus & Detritus & 0.02 \\
\hline
\end{tabular}

L: larvas; IAi \% = Índice alimentario.

L: larvae; IAi $\%=$ Food index.

bajos valores de índice alimentario $1.4 \% \mathrm{IAi}$ y $7 \times 10^{-2} \%$ IAi, respectivamente (Fig. 3). La similitud trófica entre los intervalos de tallas mostró dos grupos: el grupo A con las tallas I y III (similitud 0.62) por el consumo de insectos, y el grupo B con las tallas II, IV, V y VI (similitud 0.64) por el consumo de microcrustáceos.

Variación de la dieta entre sexos: se identificó 18 y 11 ítems en hembras y machos, respectivamente, sin diferencias significativas, dado que se observó una similitud trófica de 0.78 , esto debido a que los adultos muestran una especialización trófica sobre el consumo de Microcrustáceos que constituyó el componente alimenticio más importante en la dieta, tanto en hembras (97.2 \% IAi Copépoda y Cladócera) como en machos (80.04 \% IAi: Rotífera, Copépoda y Cladócera), seguidos por el consumo de insectos [hembras (1.43\% IAi) $v s$ machos (17.4\% IAi)].

Variación temporal de la dieta: la similitud trófica fue del 0.63 , donde los momentos de aguas altas y bajas fueron las más similares con el 0.81 , y no se observaron diferencias estadísticamente significativas $(\mathrm{K}-\mathrm{W}, \mathrm{p}=0.07)$, dado el alto porcentaje de microcrustáceos en la dieta. En aguas altas los microcrustáceos representaron el $99 \%$ IAi, le sigue insectos alóctonos (0.27\% IAi), escamas (0.32\% IAi) y detritus ( $0.11 \%$ IAi), mientras que en aguas bajas los microcrustáceos correspondieron el $100 \%$ IAi. La dieta durante la retirada de las 


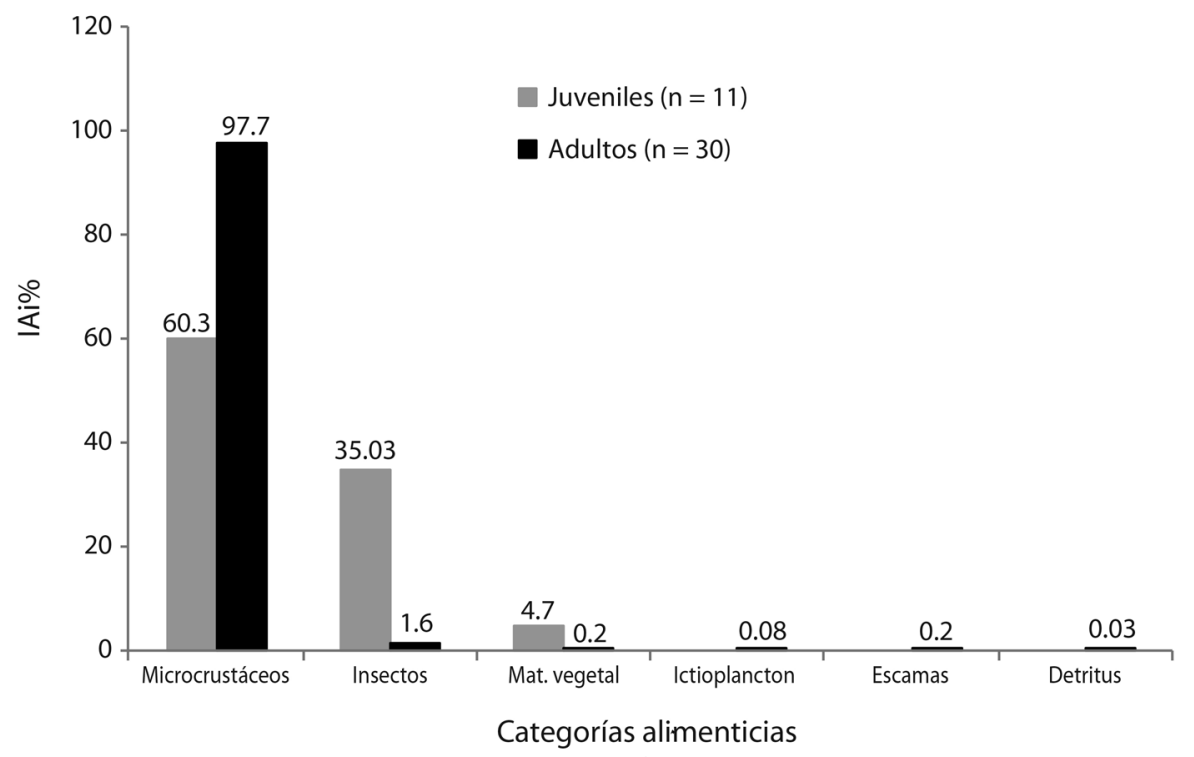

Fig. 3. Índice alimentario (IAi \%) en juveniles (34-124 mm LE) y adultos (124.1-190 mm LE). n = número de estómagos con alimento.

Fig. 3. Food index (IAi \%) in juveniles (34-124 mm LS) and adults (124.1-190 mm LS). $\mathrm{n}=$ number of stomachs with food.

aguas incluyo microcrustáceos (77.8\% IAi), ictioplancton (43\% IAi), insectos (18.3\% IAi), material vegetal $(3.67 \%$ IAi) y escamas $(0.08$ \% IAi) (Fig. 4).

Estructuras bucofaríngeas y tracto digestivo: Triportheus magdalenae posee dos hileras de dientes en el premaxillar, en la fila externa un promedio de 5 dientes $( \pm 0.5 \mathrm{SD})$, mínimo 5 y máximo 6 tricúspides; y en la fila interna 9 ( $\pm 0.6 \mathrm{SD})$, mínimo 8 y máximo 10 dientes tricúspides-multicúspides, el maxillar es adentado, y el dentario posee 7 ( $\pm 1.9 \mathrm{SD})$ dientes multicúspides, con mínimo 6 (adultos) y máximo 8 (juveniles) (Fig. 5). Los arcos branquiales no poseen lóbulos o protuberancias, y

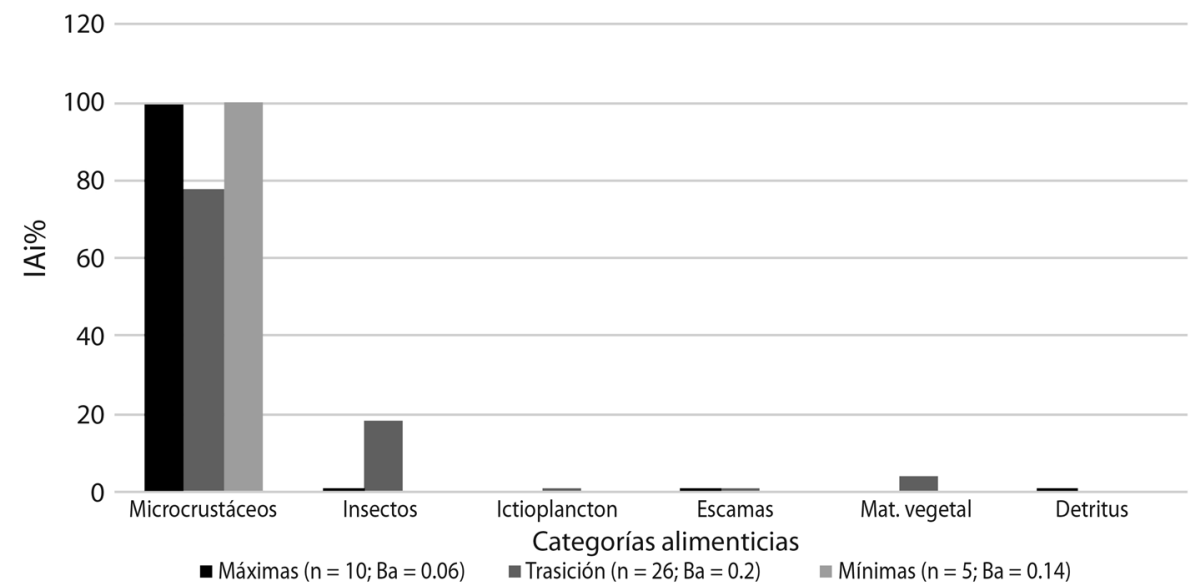

Fig. 4. Índice alimentario (IAi \%) momentos de muestreo: aguas máximas (M2); aguas en transición o en retirada (M3 y M4); aguas mínimas (M5); $\mathrm{n}$ = número de estómagos con alimento; $\mathrm{B}_{\mathrm{A}}=$ Índice estandarizado de Levin (Krebs, 2014).

Fig. 4. Food index (IAi\%) sampling moments: maximum waters (M2); waters in transition (M3 and M4); minimum water (M5); $\mathrm{n}=$ number of stomachs with food; BA = Levin's standardized index (Krebs, 2014). 

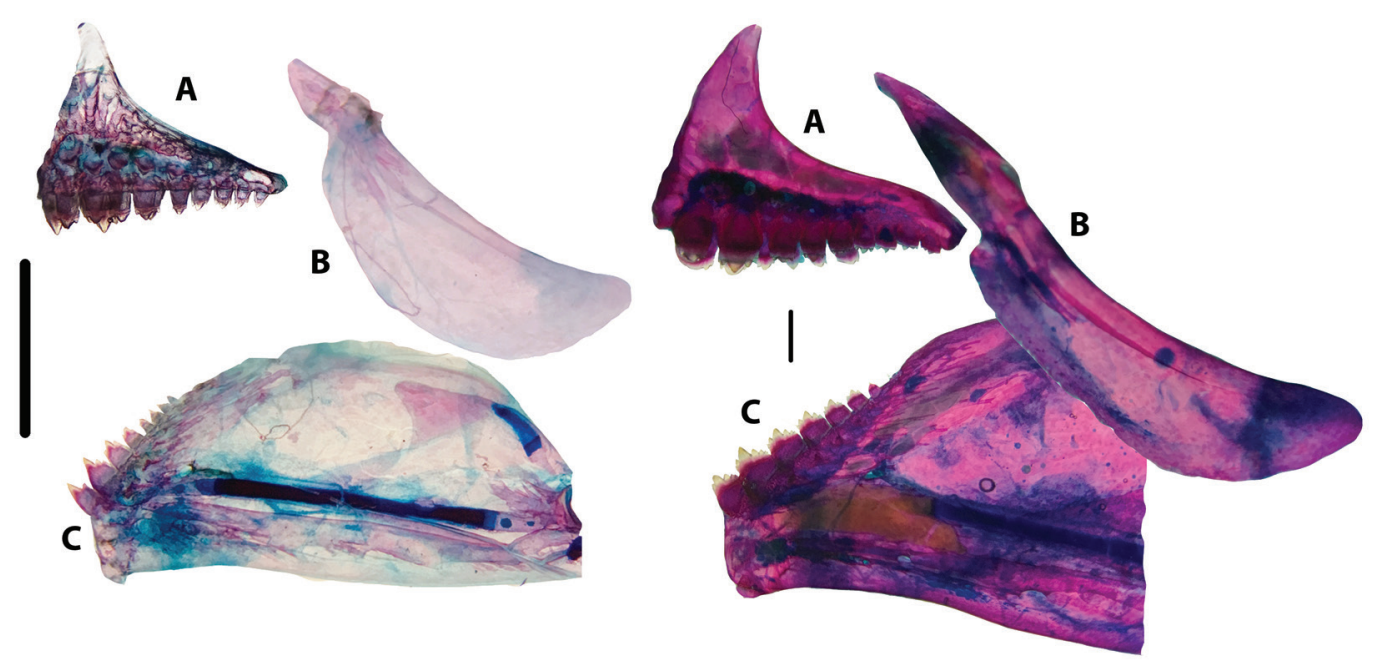

Fig. 5. Mandíbulas de T. magdalenae, Talla I izquierda y V derecha: A) pre-maxillar con dos hileras de dientes tricúspides y multicúspides; B) maxillar sin dientes; C) dentario con dientes multicúspides. Escala de $1 \mathrm{~cm}$;

Fig. 5. Jaws of T. magdalenae, size I left and V right: A) pre-maxillar with two rows of tricuspid and multicuspid teeth; B) maxillar without teeth; C) tooth with multicuspid teeth. Scale of $1 \mathrm{~cm}$.

las branquiespinas son más largas en el epibranquial, luego se acortan progresivamente hacia el hipobranquial, el número y la longitud de las branquiespinas varían significativamente (ANOVA; $p<0.001$ ), dado que aumentan en número y longitud conforme los individuos pasan de juveniles a adultos (Cuadro 4).

El estómago es de forma sacular más largo $(19.5 \mathrm{~mm})$ que ancho $(9.1 \mathrm{~mm})$ con 8 a 16 ciegos pilóricos, siendo 14 el número más

\section{CUADRO 4}

Número promedio de branquiespinas en el primer arco branquial izquierdo $\left(\# \mathrm{BE} 1^{\circ}\right)$ por cada intervalo de tallas

TABLE 4

Average number of gillrakers in the first left gill arch $\left(\# \mathrm{BE} 1^{\circ}\right)$ for each size range

\begin{tabular}{lcc} 
Tallas & LE $($ rango mm) & \#BE1 ${ }^{\circ}(\mathrm{SD})$ \\
I $(\mathrm{n}=2)$ & $38.2(34-64)$ & $58( \pm 10.9)$ \\
II $(\mathrm{n}=2)$ & $91.5(64.1-94)$ & $81( \pm 13.9)$ \\
III $(\mathrm{n}=3)$ & $109.9(94.1-124)$ & $101( \pm 5.0)$ \\
$\mathbf{I V}(\mathrm{n}=3)$ & $146.1(124.1-154)$ & $107( \pm 8.08)$ \\
$\mathbf{V ~}(\mathrm{n}=3)$ & $167.9(154.1-184)$ & $113( \pm 3.46)$ \\
$\mathbf{V I}(\mathrm{n}=3)$ & $179.7(184.1-214)$ & $108( \pm 12.2)$ \\
\hline
\end{tabular}

$\mathrm{n}=$ número de ejemplares; $\mathrm{SD}=$ desviación estándar. $\mathrm{n}=$ number of examples; $\mathrm{SD}=$ standard deviation. frecuente. El peso y la longitud del estómago estuvieron significativamente asociados con la longitud estándar (Sperman; $r=0.79 ; \mathrm{p}=2.72$ $\mathrm{x} 10^{-20}, \mathrm{y} \mathrm{r}=0.72 ; \mathrm{p}=1.54 \times 10^{-15}$, respectivamente). La longitud del intestino mostró una media de $86.3 \mathrm{~mm}( \pm 33.3)$ la cual equivale al $58.7 \%$ la longitud estándar.

Rasgos ecomorfológicos: entre los valores de los rasgos ecomorfológicos para cada intervalo de talla (I a VI) existieron diferencias significativas $(\mathrm{K}-\mathrm{W} ; \mathrm{p}<0.001)$, esto debido a el número y longitud de las branquiespinas (se excluyen las formas larvales las cuales no fueron consideradas), mientras que entre sexos no existieron diferencias significativas (ANDEVA; $\mathrm{p}=0.91)$ (Cuadro 5).

Los dos primeros ejes del ACC explicaron el $96.4 \%$ de la variación (1000 permutaciones; $\mathrm{p}=0.8)$, en el eje $\mathrm{X}=90.8 \%$ los rasgos que se correlacionaron directa y positivamente con el consumo de microcrustáceos (Cladócera y Copépoda) fueron la altura relativa de la boca (ARB), configuración de la aleta pectoral (CAP), número de branquiespinas (NBE) y longitud relativa de las branquiespinas (LRBE). Por otro lado, la explotación del 
CUADRO 5

Valores en intervalos de tallas y sexo de los rasgos ecomorfológicos considerados

TABLE 5

Values in size and sex ranges of ecomorphological traits

\begin{tabular}{|c|c|c|c|c|c|c|c|c|c|c|c|}
\hline Tallas & LE mm(mín-máx) & LRC & $\mathrm{ARB}$ & DRO & PRO & NBE & LRBE & LRI & CAP & PB & $\mathrm{D}$ \\
\hline $\mathbf{I}(\mathrm{n}=2)$ & $38.2(34-64)$ & 0.28 & 0.103 & 0.066 & 0.607 & 58 & 8.4 & 0.398 & 0.143 & 2 & 1 \\
\hline II $(\mathrm{n}=2)$ & $91.5(64.1-94)$ & 0.26 & 0.093 & 0.066 & 0.599 & 81 & 15.4 & 0.354 & 0.071 & 2 & 1 \\
\hline III $(\mathrm{n}=8)$ & 109. $9(94.1-124)$ & 0.26 & 0.089 & 0.066 & 0.628 & 98 & 20.3 & 0.534 & 0.057 & 2 & 1 \\
\hline $\mathbf{I V}(\mathrm{n}=32)$ & $146.1(124.1-154)$ & 0.24 & 0.079 & 0.059 & 0.636 & 107 & 24.8 & 0.556 & 0.042 & 2 & 1 \\
\hline $\mathbf{V}(\mathrm{n}=40)$ & $167.9(154.1-184)$ & 0.24 & 0.084 & 0.055 & 0.638 & 113 & 29.3 & 0.652 & 0.035 & 2 & 1 \\
\hline $\mathbf{V I}(\mathrm{n}=5)$ & $179.7(184.1-214)$ & 0.23 & 0.074 & 0.053 & 0.657 & 108 & 31.2 & 0.657 & 0.033 & 2 & 1 \\
\hline$\widehat{\jmath}(n=29)$ & $146.9(114-170)$ & 0.235 & 0.078 & $*$ & 0.634 & $*$ & $*$ & 0.581 & 0.007 & 2 & * \\
\hline ㅇ $(\mathrm{n}=32)$ & $166.9(130-190)$ & 0.243 & 0.085 & $*$ & 0.631 & $*$ & $*$ & 0.645 & 0.036 & 2 & $*$ \\
\hline
\end{tabular}

$\mathrm{n}$ = número de individuos medidos; mín = mínimo; máx = máximo: $\mathrm{LRC}=$ longitud relativa de la cabeza, $\mathrm{ARB}=$ altura relativa de la boca; $\mathrm{PB}=$ posición de la boca $[2=$ terminal $] ; \mathrm{DRO}=$ diámetro relativa del ojo; $\mathrm{PRO}=$ posición relativa de los ojos; $\mathrm{D}=$ dientes $[1=$ pocos dientes $(<50)$ de forma cónica o forma multicuspidal $]$; $\mathrm{NBE}=$ número de branquiespinas en el primer arco branquial izquierdo; LRBE = longitud relativa de branquiespinas en el primer arco branquial izquierdo; LRI = longitud relativo del intestino; $\mathrm{CAP}=$ configuración de la aleta pectoral izquierda; $*=$ sin calculo.

$\mathrm{n}=\#$ of individuals measured. $\mathrm{LRC}=$ relative head length, $\mathrm{ARB}=$ relative height of the mouth; $\mathrm{PB}=$ position of the mouth $[2=$ terminal $] ; \mathrm{DRO}=$ relative eye diameter; $\mathrm{PRO}=$ relative position of the eyes; $\mathrm{D}=$ teeth $[1=$ few teeth $(<50)$ of conical or multicuspidal shape]; NBE = number of gillrakers in the first left branchial arch; LRBE $=$ relative length of gillrakers in the first left branchial arch; LRI = relative length of the intestine; $\mathrm{CAP}=$ configuration of the left pectoral-fin; $*=$ no calculation.

consumo de otros componentes como las algas fitoplanctónicas explicadas en el eje $\mathrm{Y}=5.6$ estuvo correlacionada con la longitud relativa del intestino (LRI) y la posición relativa de los ojos (PRO) (Fig. 6).

\section{DISCUSIÓN}

El hábito zooplanctófago de la especie concuerda con lo encontrado en otros trabajos como Rodríguez \& Rodríguez (1976) los cuales concluyeron que en ecosistemas lénticos de la región alta del Canal del Dique sigue un régimen zooplanctófago, ayudada por sus numerosas y largas branquiespinas que constituyen el principal indicio del tipo de alimentación. Moreno (2006) en la ciénaga de Ayapel también la reporta con hábito trófico zooplanctófago, igual que otros trabajos no publicados como Benavides (2007) en el embalse El Guájaro donde los análisis correspondieron a ejemplares entre 140 y $260 \mathrm{~mm} \mathrm{LT}$, por lo tanto, solo comparables con los adultos del presente estudio. Sin embargo, Herrera-Molina (2010) indicó que aunque la especie tiene hábitos zooplanctófagos, también tiene la capacidad de cambiar la dieta dependiendo de la oferta del recurso, ya que puede consumir insectos y restos de material vegetal alóctonos en ambientes lóticos, igual a lo observado en este estudio.

Generalmente las especies del género Triportheus tienen hábitos tróficos omnívoros con tendencia a la insectivoría ( $T$. albus, $T$. elongatus, T. paranensis, T. nematurus, T. signatus y $T$. angulatus), donde pueden presentar diferencias en la composición de sus dietas dependiendo de la oferta alimentaria en el tipo de ecosistema habitado (Galina \& Hahn, 2003). Por otro lado, T. culter especie hermana de $T$. magdalenae (Mariguela et al. 2016) es exclusivamente zooplanctófaga independientemente del tipo de ecosistema en el que se encuentre (Galina \& Hahn, 2003; Alván-Aguilar, Alencar, Hardy, \& Chu-Koo, 2009).

Triportheus magdalenae posee dientes tricúspides y multicúspides que están asociado a morder o desgarrar (Gatz, 1979). En el estudio de Rodríguez \& Rodríguez (1976) describieron 


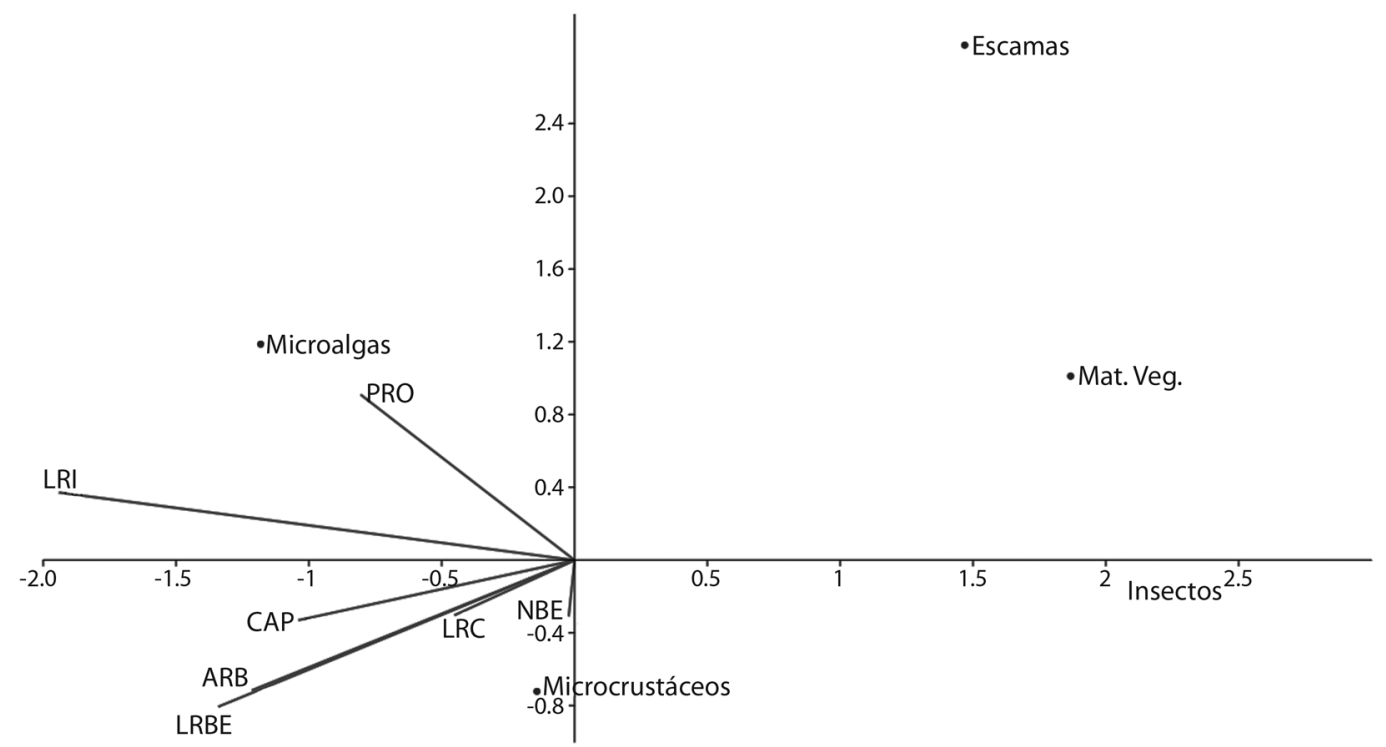

Fig. 6. Análisis de Correlación Canónica (ACC) entre la dieta y rasgos ecomorfológicos. Los ejes X y el eje Y explican el $96.4 \%$ de la variación; las abreviaciones siguen cuadro 1. El círculo punteado indica los rasgos asociados con el consumo de microcrustáceos.

Fig. 6. Canonical Correlation Analysis (CCA) between diet and morphological traits. The X and Y axes explain 96.4\% of the variation; the abbreviations follow table 1 . The dotted circle indicates the traits associated with the consumption of microcrustaceans.

en el premaxilar dos filas de dientes (10 en la fila externa, y 14 en la interna), el maxilar adentado, y en el dentario 14 dientes. En comparación con otras especies del mismo género, posee un menor número de dientes, por ejemplo, Almeida (1984) reportó en $T$. culter dos filas de dientes en el premaxilar (12 tricúspides en la fila externa y 19 multicúspides en la fila interna), con 16 dientes multicúspides en el dentario, y el maxilar adentado como T. magdalenae.

Las branquiespinas y el tipo de dientes son consideradas como las estructuras que están más íntimamente relacionadas con el tipo de alimento ingerido para las especies del género Triportheus (Almeida, 1984; Galina \& Hahn, 2003). Por ejemplo, T. culter poseen 109 branquiespinas, y mayor longitud de las branquiespinas en comparación con $T$. angulatus y T. elongatus con valores medios de 51 y 28, respectivamente. Para T. paranensis la explotación del zooplancton se debe a una especialización de los arcos branquiales, que son más delgados, largos y unidos que las de sus congéneres, dichas modificaciones en el aparato branquial, les permiten aumentar la eficiencia de la captura del zooplancton (Almeida, 1984; Galina \& Hahn, 2003). Los resultados de este trabajo mostraron que las variables relacionadas positivamente con la actividad de filtración y el tipo de hábito trófico zooplanctófago de $T$. magdalenae fueron el número y longitud de las branquiespinas, donde se evidencio variación en el número entre los juveniles y los adultos, con valores que oscilaron entre 58 y 108 branquiespinas, respectivamente, indicando entonces que conforme los individuos crecen aumentan el número y longitud relativa de las branquiespinas, lo cual está relacionado con cambios en la dieta, con un consumo de zooplancton e insectos en juveniles a un consumo predominate de zooplancton en adultos.

\section{AGRADECIMIENTOS}

Al programa de Maestría en Biología y Grupo de Investigación Biodiversidad del 
Caribe Colombiano de la Universidad del Atlántico, a la Vicerrectoría de investigaciones y la Facultad de Ciencias Básicas por el apoyo logístico y financiero con el proyecto de "Impacto Caribe", y la beca del Consejo Profesional de Biología (convocatoria CPBiol.2015). A la gobernación del Atlántico por parte de la financiación en marco del proyecto regalías: "Estado actual de los recursos hidrobiológicos de las ciénagas del Sur del Atlántico y subregión canal del Dique" código 0103*2013*000009. A Donald C Taphorn por sus aportes en el abstract.

\section{RESUMEN}

Este estudio integró las características ecomorfológicas y la ecología trófica de Triportheus magdalenae en el embalse El Guájaro, Atlántico, bajo Magdalena, Colombia, a partir del análisis del contenido estomacal y rasgos ecomorfológicos. Se realizaron cinco muestreos entre septiembre 2014 y febrero 2016 para abarcar cada momento del pulso de inundación. La recolecta de los peces fue a través de artes de pesca tradicional como atarrayas y redes de arrastre. Se utilizó el índice alimentario (IAi \%) para establecer los hábitos alimentarios de la especie, y la variación de la dieta a nivel interespecífico, y se usó el coeficiente de vacuidad (CV \%). Se emplearon los índices de Levins Estandarizado por Hurlbert $\left(\mathrm{B}_{\mathrm{A}}\right)$ e índice de Morisita Simplificado para evaluar la amplitud y similitud trófica entre tallas, sexo y momentos hidrológicos. Se caracterizó las estructuras bucofaríngeas y el tracto digestivo, que junto con medidas morfológicas permitieron establecer y analizar 11 rasgos ecomorfológicos relacionados con el uso del hábitat como posición en la columna de agua y la explotación del recurso trófico. Se analizó la relación entre los rasgos ecomorfológicos con la dieta de la especie, por medio de un análisis de correspondencia canónica (ACC). Se analizaron 89 ejemplares (34-190 mm LE). T. magdalenae fue catalogado como carnívoro-zooplanctófago, con un estrecho nicho trófico $\left(\mathrm{B}_{\mathrm{A}}=0.12\right)$. Se evidenció una alta explotación de los organismos del zooplancton (95.9 IAi \%), intensivamente Cladócera (Ceriodaphnia sp.) y Copépoda (Notodiaptomus sp. y Thermocyclops sp), los juveniles (34-190 mm LE) consumieron intensamente los insectos alóctonos (3.4 IAi \%). Los rasgos que más aportan a la especialización trófica fueron la longitud relativa de las branquiespinas y el número de branquiespinas, los cuales varían significativamente entre tallas, ya que aumentan en número como en longitud conforme los individuos crecen. El coeficiente de vacuidad fue alto del $54 \%$, lo cual pudo estar asociado con la disminución en la densidad de las poblaciones de zooplancton, especialmente durante el momento de aguas mínimas fuertemente influenciado por el evento El Niño, sin embargo la dieta observada (22 ítems alimentarios) correspondió al $80 \%$ de la dieta estimada (28 ítems alimentarios estimados).

Palabras clave: dieta; rasgos ecomorfológicos; pez endémico; diversidad.

\section{REFERENCIAS}

Abelha, F., Agostinho, A., \& Goulart, E. (2001). Plasticidade trófica em peixes de água doce. Acta Scientiarum, 23(2), 425-434.

Alván-Aguilar, M. A., Alencar, Y. B., Hardy, E. R., \& ChuKoo, F. (2009). Alimentação de juvenis da sardinha papuda Triportheus flavus (Osteichthyes, Characidae) no periodo de cheia, no lago Camaleão (Amazonas, Brasil). Instituto de Investigaciones de la Amazonía Peruana. Folia Amazónica, 18(1-2), 67-73.

Almeida, G., R., (1984). Biología alimentar de tres especies de Triportheus (pisces: characoideil; characidae) do lago do Castanho, Amazonas. Acta amazónica, 14(1-2), 48-76.

Balon, E. K., Crawford, S. S., \& Lelek, A. (1986). Fish communities of the upper Danube river (Germany, Austria) prior to the new Rhein-Main-Donau connection. Environmental Biology of Fishes, 15(4), 243-271.

Benavides, L. R. (2007). Evaluación del contenido estomacal de la arenca Triportheus magdalenae Steindachner, 1878, (Pisces: Characidae) en el embalse de El Guájaro (Departamento del Atlántico) (Trabajo de grado). Universidad del Atlántico, Colombia.

Caraballo, P. (2009). Efecto de tilapia Oreochromis niloticus sobre la producción pesquera del embalse El Guájaro Atlántico-Colombia. Revista MVZ Córdoba, 14(3), 1796-1802.

Chen, L. (2002). Post-settlement diet shift of Chlorurus sordidus and Scarus schlegeli (Pisces: Scaridae). Zoological Studies, 41(1), 47-58.

CRA, Ministerio del Medio Ambiente, Banco Interamericano de Desarrollo, \& CARDIQUE. (2002). Plan de Manejo Ambiental del complejo de ciénagas El Totumo, El Guájaro, y El Jobo en la ecorregión estratégica del Canal del Dique (Convenio No. 201680), Recuperado de: http://www.crautonoma.gov.co/ documentos/recursohidrico/6_Diagnóstico\%20 Ordenamiento $\% 20$

Domínguez, E., \& Fernández, H. (Eds.). (2009). Macroinvertebrados bentónicos sudamericanos: sistemática y biología. Tucumán, Argentina: Fundación Miguel Lillo.

Ebert, D., \& Bizarro, J. (2007). Standardized diet compositions and trophic levels of skates (Chondrichthyes: 
Rajiformes: Rajoidei). Environmental Biology of Fishes, 80(2), 221-237. doi: 10.1007/s10641-007-9227-4

Fernández, E. M., Ferriz, R. A., Bentos, C. A., \& López, G. R. (2012). Dieta y ecomorfología de la ictiofauna del arroyo Manantiales, provincia de Buenos Aires, Argentina. Revista del Museo Argentino de Ciencias Naturales nueva serie, 14(1), 1-13.

Fernando, C. H. (2002). A Guide to Tropical freshwater Zooplankton: Identification, Ecology and Impact on Fisheries. Netherlands: Backhuys Publisher, Leiden.

Galina, A. B., \& Hahn S. N. (2003). Comparação da dieta de duas espécies de Triportheus (Characidae, Triportheinae), em trechos do reservatório de Manso e lagoas do río Cuiabá, Estado do Mato Grosso. Acta Scientiarum Biological Sciences, 25(2), 345-352.

García-Alzate, C. A., Román-Valencia, C., \& Ana M. Barrero. (2012). Biología alimentaria y reproductiva de Farlowella vittata (Siluriformes: Loricariidae) en la cuenca del río Güejar, Orinoquía, Colombia. Revista de Biología Tropical, 60(4), 1873-1888.

García-Alzate, C. A., Gutiérrez L. C., \& De la Parra, A. (2016). Embalse de El Guájaro: Diagnostico ambiental y estrategias de rehabilitación. En M. Alvarado (Ed.), Sur del Atlántico (pp. 148-181). Barranquilla, Colombia: Fundación Promigas. ISBN: 978-958-876

Gatz, A. J. (1979). Ecological morphology of freshwater stream fishes. Tulane Studies in Zoology and Botany, 21(2), 91-124.

Hammer, Ø. (2016). Paleontological Statistics (PAST Version 3.14). Natural History Museum, University of Oslo. Retrieved from http://folk.uio.no/ohammer/past

Herrera-Molina, C. A. (2010). Ecología trófica y reproductiva de la arenca Triportheus magdalenae y la sardina Astyanax magdalenae en el sistema del río Sogamoso, Colombia. (Trabajo de grado). Universidad de Nacional de Colombia.

Hyslop, E. J. (1980). Stomach contents analysis, a review of methods and their application. Journal of Fish Biology, 17, 411-429.

Hugueny, B., \& Pouilly, M. (1999). Morphological correlates of diet in an assemblage of West African freshwater fishes. Journal of Fish Biology, 54, 1310-1325.

Ibarra-Trujillo, E. J., \& García-Alzate, C. A. (2017). Ecología trófica y reproductiva de Hemibrycon sierraensis (Characiformes: Characidae), pez endémico del río Gaira, Sierra Nevada de Santa Marta, Colombia. Revista de Biología Tropical, 65(3), 1033-1045.

Jiménez-Segura, L. F., Carvajal-Quintero, J., \& Aguirre, N. (2010). Las ciénagas como hábitat para los peces: estudio de caso de la ciénaga de Ayapel (Córdoba), Colombia. Actualidades Biológicas, 32(92), 53-64.
Kawakami, E., \& Vazzoler, G. (1980). Método gráfico e estimativa de índice alimentar aplicado no estudo de alimentação de peixes. Boletín del Instituto Oceanográfico de Venezuela, 29(2), 205-207.

Krebs, C. J. (2014). Ecological Methodology. Menlo Park California: Addison-Welsey Educational Publisher, Inc.

Malabarba, M. (2004). Revision of the Neotropical genus Triportheus Cope, 1872 (Characiformes: Characidae). Neotropical Ichtyology, 2(4), 167-204.

Mariguela, T. C., Roxo, F. F., Foresti, F., \& Oliveira, C. (2016). Phylogeny and biogeography of Triportheidae (Teleostei: Characiformes) based on molecular data. Molecular Phylogenetics and Evolution, 96, 130-139.

Mérigoux, S., \& Ponton, D. (1998). Body shape, diet and ontogenetic diet shifts in young fish of the Sinnamary River, French Guiana, South America. Journal of Fish Biology, 52, 556-569.

Morales, J., \& García-Alzate, C. A. (2016). Estructura trófica de los peces en arroyos del Corral de San Luis, cuenca del Bajo Magdalena, Caribe, Colombia. Revista de Biología Tropical, 64(2),715-732.

Moreno, G. (2006). Ecología básica de Triportheus magdalenae y Pimelodus clarias en la ciénaga de Ayapel, (cuenca media del río San Jorge) durante el ciclo hidrológico 2004-2005 (Trabajo de grado). Universidad de Antioquia, Medellín.

Pianka, E. R. (2000). Evolutionary Ecology (6th Ed.). San Francisco: Addison Wesley Longman.

Pouilly, M., Lino, F., Bretenoux, J. G., \& Rosales, C. (2003). Dietary-morphological relationships in a fish assemblage of the Bolivian Amazonian floodplain. Journal of Fish Biology, 62, 1137-1158.

Ramírez, J. (2000). Fitoplancton de agua dulce: aspectos ecológicos, taxonómicos y sanitarios. Colombia: Editorial Universidad de Antioquia.

Ramírez, F., Lee, T., \& Mojica, I. (2015). Dietary-morphological relationships of nineteen fish species from an Amazonian terra firme blackwater stream in Colombia. Limnologica, 52, 89-102.

Rodríguez, G., \& Rodríguez, B. (1976). Contribución al estudio bioecológico de la "Arenca" Triportheus magdalenae Steindachner 1878. Instituto de desarrollo de los Recursos Naturales Renovables.

Roldán, G. (1996). Guía para el estudio de los macroinvertebrados acuáticos del Departamento de Antioquia. Bogotá Colombia: Impreades Presencia S.A.

Sánchez, R., Galvis, G., \& Victoriano, P. F. (2003). Relaciones entre características del tracto digestivo y los 
hábitos alimenticios de peces del río Yucao, sistema del río Meta (Colombia). Gayana, 67(1), 75-86.

Taylor, W. R. (1969). An enzyme method of clearing and staining small vertebrales. Procedings of the United Sates Natural Museum, 122(3596) 1-17.

Teixeira, I \& Bennemann, S. (2007). Ecomorfologia refletindo a dieta dos peixes em um reservatório no sul do Brasil. Biota Neotropica, 7(2), 67-76.

Ter-Braak, C. (1986). Canonical correspondence analysis: a new eigenvector technique for 540 multivariate direct gradient analysis. Ecology, 67, 1167-1179.

Valverde, T., Meave, J. A., Carabias, J., \& Cano-Santana, Z. (2005). Ecología y medio ambiente. México: Pearson Educación.

Villalón, J., \& Vega, A. (2016). Aspectos históricos, Sur del Atlántico: cuatro mil años de historia. En M. Alvarado (Ed.), Sur del Atlántico (pp. 29-49).
Barranquilla, Colombia: Fundación Promigas. ISBN: 978-958-8767-43-7

Watson, D., \& Balon, E. (1984). Ecomorphological analysis of fish taxocenoses in rainforest stream of northern Borneo. Journal of Fish Biology, 145(1), 303-320.

Winemiller, K. O. (1991). Ecomorphological diversification in low-land fresh water fish assemblages from five biotic regions. Ecological Monographs, 61, 343-365.

Zarate-Hernández, R., Aguirre-León, A., Ortiz-Burgos, S., \& Castillo-Rivera, M. (2007). Ecomorfología de peces estuarinos del Golfo de México. ContactoS, $66,12-20$.

Zhao, T., Villéger, S., Lek, S., \& Cucherousset, J. (2014). High intraspecific variability in the functional niche of a predator is associated with ontogenetic shift and individual specialization. Ecology and Evolution, 4(24), 4649-4657. 